\title{
A Newborn Admitted with Hyponatremia and Hyperkalemia Clinic and Diagnosed with Primary Hypoaldosteronism
}

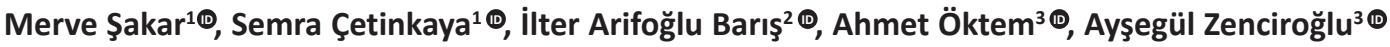 \\ Rüveyde Bundak ${ }^{4}$, Şenay Savaş Erdeve ${ }^{1 \oplus}$
}

${ }^{1}$ Department of Pediatric Endocrinology, Health Sciences University, Dr Sami Ulus Obstetrics and Gynecology, Child Health and Diseases Training and Research Hospital, Ankara, Turkey

2 Department of Neonatology, Dr. Burhan Nalbantoğlu Public Hospital, Lefkoşa, Cyprus

${ }^{3}$ Department of Neonatology, Health Sciences University, Dr Sami Ulus Obstetrics and Gynecology, Child Health and Diseases Training and Research Hospital, Ankara, Turkey

${ }^{4}$ Department of Pediatric Endocrinology, University of Kyrenia, Lefkoşa, Cyprus

Cite as: Şakar $M$, Çetinkaya $S$, Arifoğlu Barış i, Öktem A, Zenciroğlu A, Bundak R, Savaş Erdeve Ş. A newborn admitted with hyponatremia and hyperkalemia clinic and diagnosed with primary hypoaldosteronism. Trends in Pediatrics 2021;2(2):94-8.

Received: 02 December 2020

Accepted: 26 February 2021

Publication date: 29 June 2021

Keywords: CYP11B2, newborn, primary hypoaldosteronism, salt-wasting

\section{Merve Şakar}

Department of Pediatric Endocrinology, Health Sciences University, Dr Sami Ulus Obstetrics and Gynecology, Child Health and Diseases Training and Research Hospital, Ankara, Turkey

ORCID: 0000-0002-4960-6092

mervesakar_capa@hotmail.com

S. Çetinkaya 0000-0003-3974-2872

i. Arifoğlu Barıș 0000-0001-6254-751X

A. Öktem 0000-0001-7209-6732

A. Zenciroğlu 0000-0002-3488-4962

R. Bundak 0000-0001-5097-6448

Ş. Savaş Erdeve 0000-0002-4164-5089

\begin{abstract}
Primary hypoaldosteronism is a rare autosomal recessive disorder that causes defects in aldosterone synthase enzyme activity due to an inactivating mutation in the CYP11B2 gene. Patients with primary hypoaldosteronism exhibit symptoms such as vomiting, dehydration, feeding problems, and failure to thrive. This disease is characterized by hyponatremia, hyperkalemia, metabolic acidosis, high renin, and low aldosterone levels. It also causes salt loss syndrome in the newborn period. In this article, we described a newborn with primary hypoaldosteronism who showed feeding problems and weight loss. Laboratory findings revealed hyponatremia, hyperkalemia, high plasma renin, and low aldosterone levels, while genetic analysis showed homozygous c.788T $>A$ (p.Ile263Asn) variant in the CYP11B2 gene (NM_000498.3). Our patient responded well to oral salt and fludrocortisone treatments. Early diagnosis and treatment are particularly important because primary hypoaldosteronism causes life-threatening electrolyte disorders and salt loss syndrome.
\end{abstract}

\section{INTRODUCTION}

Aldosterone is a mineralocorticoid hormone that is synthesized only in the zona glomerulosa of the adrenal cortex. By acting on the distal renal tubules and cortical collecting ducts in the kidney, it increases the absorption of sodium and water from the lumen and ensures the excretion of potassium and hydrogen ions into the lumen. The aldosterone synthase enzyme is one of the Cytochrome-P450 enzymes encoded by the CYP11B2 gene, which plays a role in the last three steps of aldosterone biosynthesis. It provides the conversion of 11-deoxycorticosterone (DOC) to corticosterone, the conversion of corticosterone to 18-hydroxy-corticosterone (18-OHB), and finally the synthesis of aldosterone. ${ }^{1}$ 
Primary hypoaldosteronism occurs because of a defect in the aldosterone synthase enzyme that is developed due to an inactivating mutation in the CYP11B2 gene. It is a rare inherited disorder transmitted as either an autosomal recessive or autosomal dominant trait with mixed penetrance. Its incidence is below $1: 1,000,000 .^{2}$ There are two types of aldosterone synthase deficiency (ASD) based on the specific defects in aldosterone synthesis: type 1 and type 2. These two types of ASD have similar clinical features. In both conditions, aldosterone cannot be synthesized, and plasma renin activity is elevated. In type 1 ASD, the level of 18-hydroxycorticosterone (18-OHB) is also decreased due to the lack of enzyme activity. On the other hand, in type 2 ASD, the 18-hydroxy-corticosterone (18-OHB) level is increased due to residual enzyme activity. ${ }^{3}$ ASD leads to neonatal salt loss syndrome and life-threatening electrolyte imbalances. Clinical symptoms are salt loss, vomiting, dehydration, feeding problems, and developmental delay. It is also characterized by hyponatremia, hyperkalemia, metabolic acidosis, high renin, and low aldosterone levels. ${ }^{1}$

Primary hypoaldosteronism is an exceedingly rare disease. To our knowledge, only 45 cases have been reported in the literature so far. ${ }^{4}$ The fact that the clinical course of the disease improves over time may be related to small number of cases. This article presents a case study and a literature discussion about a newborn diagnosed with primary hypoaldosteronism and exhibiting feeding problems and weight loss.

\section{CASE REPORT}

Our female patient with a birth weight of $2230 \mathrm{~g}$ was delivered normally after 36 weeks of gestation in Cyprus from a primiparous woman. Her parents were Turkish and non-consanguineous. After birth, she was admitted to the intensive care unit because of respiratory distress, which improved after she received oxygen therapy for two days. Feeding problems and weight loss developed on the seventh day.

Upon follow-up, on physical examination her body weight $(2100 \mathrm{~g})$, her height $(51 \mathrm{~cm})$, her heart rate (165bpm), and her blood pressure were (85/42
$\mathrm{mmHg}$ ) measured. She had a normal female phenotype without any genital anomaly and skin hyperpigmentation. Laboratory examinations showed that her serum sodium level was significantly low (123 mEq/L: normal range 132-147), while her serum potassium level was significantly high (8.8 $\mathrm{mEq} / \mathrm{L}$ : normal range 3.6-6.1). Her serum glucose level was within normal limits (92 mg/dl), and there was no metabolic acidosis or alkalosis. Her urine test was normal. Abdominal ultrasonography revealed no abnormality or obstruction in her kidneys or urinary tract.

IV sodium supplementation was started for hyponatremia, and calcium gluconate infusion, sodium bicarbonate infusion, salbutamol nebule, and anti-potassium treatments were started for the treatment of hyperkalemia. Fludrocortisone was started at a dose of $0.05 \mathrm{mg} /$ day, and the dose was gradually increased to $0.2 \mathrm{mg} /$ day due to continued electrolyte disturbances during follow-up. Since her electrolyte imbalance did not improve despite these treatments, she was referred to our hospital at the age of 27 days with a serum sodium level of 135 $\mathrm{mEq} / \mathrm{L}$ and a serum potassium level of $6.5 \mathrm{mEq} / \mathrm{L}$.

The hormonal tests performed for diagnosis revealed that her plasma renin activity was markedly elevated with $>100 \mathrm{ng} / \mathrm{ml} / \mathrm{h}$ (normal range 2.4-37 ng/ml/h), while her aldosterone level was low with $11.6 \mathrm{ng} / \mathrm{dl}$ (normal range 19-141 ng/dl). The results of hormonal tests were as follows: serum adrenocorticotropic hormone (ACTH): $8.2 \mathrm{pg} / \mathrm{ml}$ (normal range 0-46), cortisol $(9.7 \mu \mathrm{g} / \mathrm{dl}$ (normal range 5.5-22.0), 17-OHprogesterone (17-OHP) $0.37 \mathrm{ng} / \mathrm{ml}$ (normal range 0-6.3), and dehydroepiandrosterone sulfate (DHEA$\left.\mathrm{SO}_{4}\right): 4759 \mu \mathrm{g} / \mathrm{dl}$.

In terms of salt loss and hyperkalemia, the diagnosis of congenital adrenal hyperplasia was excluded due to normal cortisol and adrenal androgen levels and the lack of virilization findings. The patient with high renin and low aldosterone levels was diagnosed with primary hypoaldosteronism. In the genetic analysis performed to confirm the diagnosis, the homozygous c.788T >A (p.lle263Asn) variant was detected in the CYP11B2 gene (NM_000498.3). Sequence analysis of the CYP11B2 gene was performed using a nextgeneration sequencing platform (Illumina MiSeq) with 


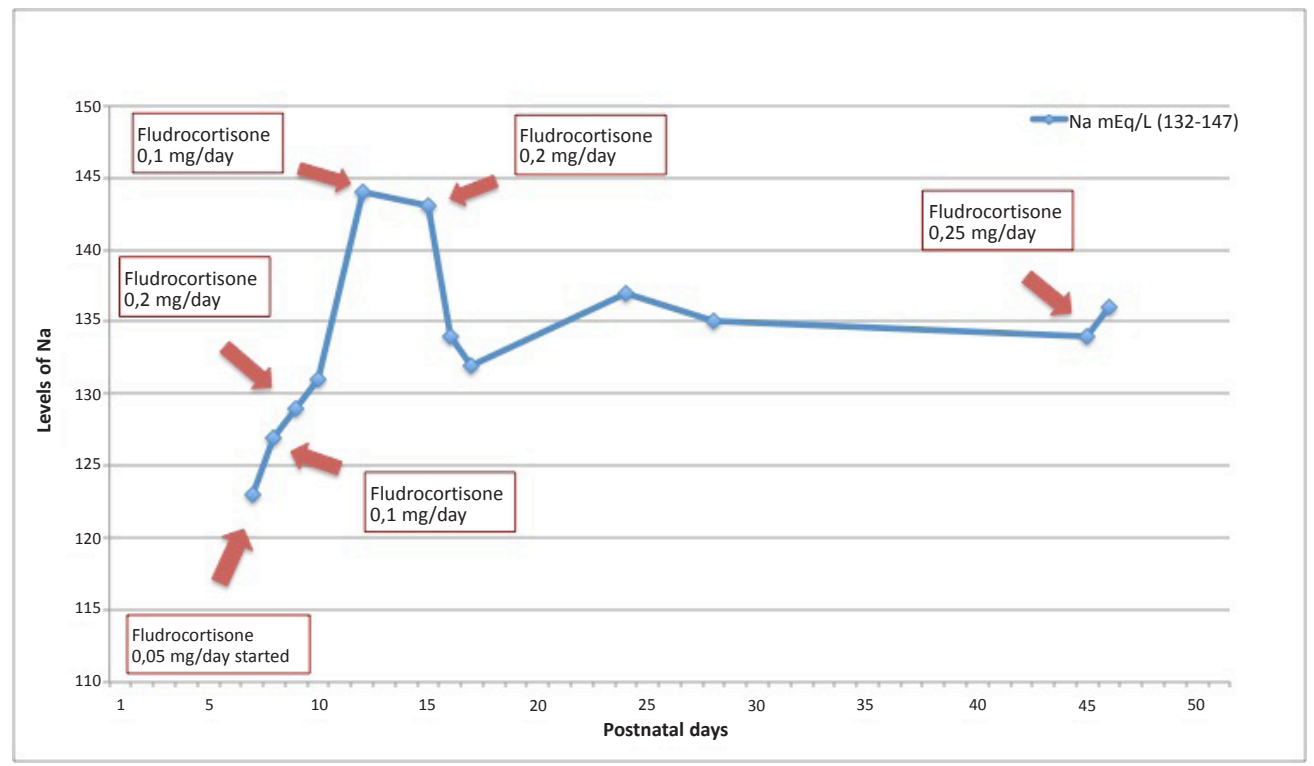

Figure 1. Serum sodium concentrations during the fludrocortisone treatment

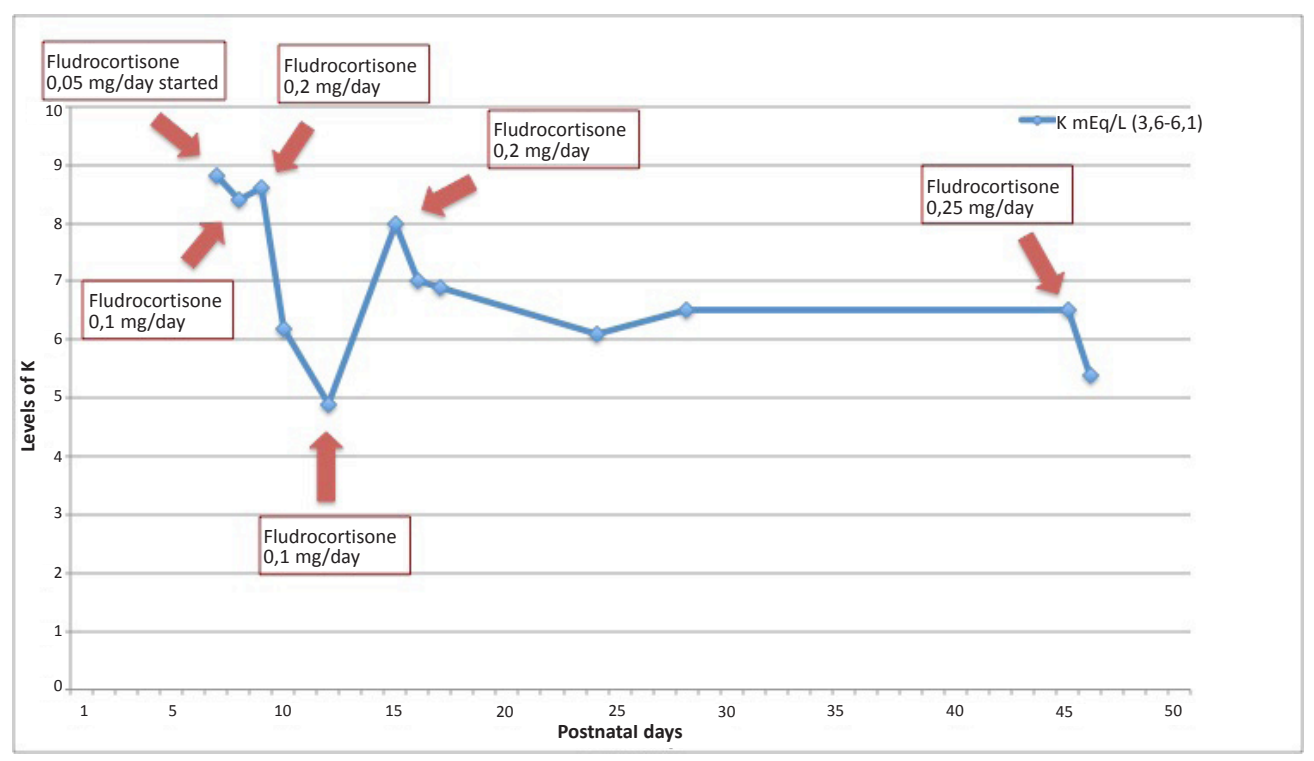

Figure 2. Serum potassium concentrations during the fludrocortisone treatment

PCR-based library preparation. This variant is classified as pathogenic according to the ACMG criteria and its presence in this disease has been previously reported in the literature. The treatment plan of the patient involved daily oral doses of $0.25 \mathrm{mg}$ fludrocortisone and $1 \mathrm{~g} \mathrm{Na} \mathrm{CL}$ supplement. The electrolyte levels returned to normal afterward (Figures 1 and 2).

We continued to follow up our patient in Cyprus, where she received oral doses of $0.1 \mathrm{mg}$ of fludrocortisone and $3 \mathrm{~g}$ salt per day. After 15 months, her body weight was $10.5 \mathrm{~kg}$, and her height was 77 $\mathrm{cm}$. The last laboratory tests revealed that her serum sodium level was $137 \mathrm{mEq} / \mathrm{L}$ while her serum potassium level was $4.1 \mathrm{mEq} / \mathrm{L}$.

\section{DISCUSSION}

In this article, we described a newborn patient with feeding problems and an inability to gain weight. 
Hyponatremia, hyperkalemia, high renin, and low aldosterone levels were found, and further genetic testing confirmed that the patient had primary hypoaldosteronism.

Primary hypoaldosteronism typically causes vomiting, signs of dehydration, hypovolemia, and failure to thrive in the first weeks of life. The biochemical assessment revealed hyponatremia, hyperkalemia, increased plasma renin activity, and low aldosterone levels. ${ }^{4}$ In rare circumstances, the disease occurs among children and adults with milder phenotypic characteristics. Few cases of normal potassium levels and hyponatremia have been reported in the literature. ${ }^{5}$ Our patient exhibited nutritional problems and weight loss on the seventh day. Her serum sodium level was extremely low, and serum potassium level was extremely high, plasma renin activity increased, and aldosterone level decreased. Congenital adrenal hyperplasia (CAH) should be considered in the differential diagnosis of cases with neonatal salt wasting. The presence of genital abnormalities and high adrenal androgen levels guide the diagnosis. In our case, $\mathrm{CAH}$ was not considered because there were no signs of virilization and hyperpigmentation. Moreover, cortisol and adrenal androgen levels were all within normal limits. Fludrocortisone treatment was initiated considering primary hypoaldosteronism because of the high renin and low aldosterone levels. Our patient responded well to the fludrocortisone treatment, and improvement was achieved in electrolyte values.

Patients respond well to fludrocortisone therapy, and salt replacement is often needed in the first 1-2 years of life. The recommended starting dose for oral fludrocortisone therapy is $0.1-0.3 \mathrm{mg} /$ day. $^{3}$ During follow-up, the patient's dose should be adjusted according to parameters such as blood pressure, serum sodium and potassium levels, and plasma renin activity. ${ }^{6}$ The disease is most severe during infancy, and clinical signs improve with age. Adolescents and adults are generally asymptomatic and do not require mineralocorticoid replacement therapy. ${ }^{4}$ It is thought that this may be due to the increase in mineralocorticoid receptors and salt intake with age, extra-adrenal compensatory mechanisms, and alternative ACTH-dependent pathways in mineralocorticoid synthesis. ${ }^{7,8}$ In our case,oral fludrocortisone treatment was started at $0.05 \mathrm{mg} /$ day and increased up to $0.25 \mathrm{mg} /$ day during follow-up. An oral sodium supplement was added at a daily dose of $1 \mathrm{~g}$. Electrolyte values improved after the treatment.

To our knowledge, only 45 primary hypoaldosteronism cases have been reported so far in the literature. Twenty of these cases were classified as type 1, 12 of them as type 2 , and 13 of them were unclassified. In 44 of these cases, symptoms appeared in the first year of life. Growth retardation, recurrent vomiting, and dehydration have been reported to be the most common symptoms. In total, $98 \%$ of the cases had hyponatremia, $89 \%$ hyperkalemia, 91\% elevated PRA levels, and $67 \%$ low aldosterone levels. There was no significant difference between type 1 and type 2 cases in terms of clinical findings, biochemical results, and hormone tests except for the 18-hydroxycorticosterone level. Thirty-three (73\%) cases received fludrocortisone treatment, and it was reported that symptoms improved in all cases receiving treatment. ${ }^{4}$

In the genetic analysis of our case, a homozygous c.788T> A (p.lle263Asn) variant was found in the CYP11B2 gene. In the literature, this variant was first reported by Üstyol et al., and Turan et al. detected the same variant in two of three primary hypoaldosteronism cases. To our knowledge, our case is the fourth case of the same ethnic origin but from a different family with the same pathological variant. ${ }^{5,9}$ The case reported by Üstyol et al. exhibited salt-wasting like our patient, but unlike ours, the serum potassium level of their case was normal. One of the cases reported by Turan et al. exhibited jaundice and another displayed symptoms like vomiting, growth retardation and dehydration, and hyponatremia and hyperkalemia. We detected the same symptoms in our case. A review of the literature revealed that clinical findings are similar in cases with the p.lle263Asn and the other variants. ${ }^{4}$

In conclusion, hyponatremia and hyperkalemia are life-threatening electrolyte disorders that require urgent intervention in newborns and infants. A diagnosis of primary hypoaldosteronism should be considered when increased renin levels and relatively low aldosterone levels are detected in patients where congenital adrenal hyperplasia is excluded 
from salt loss. In cases diagnosed with primary hypoaldosteronism, clinical and laboratory improvement is achieved with mineralocorticoid and salt replacement therapy.

Conflict of Interest: The authors declared no potential conflicts of interest with respect to the research, authorship, and/or publication of this article.

Funding: The authors received no financial support for the research, authorship, and/or publication of this article.

Informed Consent: Parents of the patient provided informed consent to publish the report.

\section{REFERENCES}

1. Root AW. Disorders of aldosterone synthesis, secretion, and cellular function. Curr Opin Pediatr. 2014;26:480-6. https://doi.org/10.1097/MOP.0000000000000104

2. Bizzarri C, Pedicelli S, Cappa M, Cianfarani S. Water balance and "Salt Wasting" in the first year of life: The role of aldosterone-signaling defects. Horm Res Paediatr. 2016;86:143-53. https://doi.org/10.1159/000449057

3. White PC. Aldosterone synthase deficiency and related disorders. In: Molecular and Cellular Endocrinology. 2004;217:81-7. https://doi.org/10.1016/j.mce.2003.10.013

4. Miao H, Yu Z, Lu L, et al. Analysis of novel heterozygous mutations in the CYP11B2 gene causing congenital aldosterone synthase deficiency and literature review. Steroids. 2019;150:108448.

https://doi.org/10.1016/j.steroids.2019.108448

5. Üstyol A, Atabek ME, Taylor N, Yeung MC, Chan AOK. Case report corticosterone methyl oxidase deficiency Type 1 with normokalemia in an infant. J Clin Res Pediatr Endocrinol. 2016;8:356-9.

https://doi.org/10.4274/jcrpe.2824

6. Bonfig W, Schwarz HP. Blood pressure, fludrocortisone dose, and plasma renin activity in children with classic congenital adrenal hyperplasia due to 21-hydroxylase deficiency followed from birth to 4 years of age. Clin Endocrinol (Oxf). 2014;81:871-5. https://doi.org/10.1111/cen.12498

7. Martinerie L, Viengchareun S, Delezoide AL, et al. Low renal mineralocorticoid receptor expression at birth contributes to partial aldosterone resistance in neonates. Endocrinology. 2009;150:4414-24. https://doi.org/10.1210/en.2008-1498

8. Laetitia M, Eric P, Laurence FLH, et al. Physiological partial aldosterone resistance in human newborns. Pediatr Res. 2009;66:323-8. https://doi.org/10.1203/PDR.0b013e3181b1bbec

9. Turan I, Kotan LD, Tastan M, Gurbuz F, Topaloglu AK, Yuksel B. Molecular genetic studies in a case series of isolated hypoaldosteronism due to biosynthesis defects or aldosterone resistance. Clin Endocrinol. (Oxf). 2018;88:799-805.

https://doi.org/10.1111/cen.13603 